\title{
The effect of exchange and absorption potential in the distorted wave calculation of electron impact excitation of the autoionizing state of caesium
}

\author{
Ochieng A. A. ${ }^{1}$, Okumu J. ${ }^{1}$, Singh C. S. ${ }^{*}$ \\ 1, 1* Department of Physics, Kenyatta University, P.O Box 43844-0100, Nairobi, Kenya \\ ${ }^{1^{*}}$ Corresponding Author. Email: singh.chadra@ku.ac.ke
}

Received: 10 July 2020 / Accepted: 30 September 2020 / Published online: 20 November 2020

\begin{abstract}
We have calculated the differential and integral cross sections for electron impact excitation of the lowest autoionizing level of caesium atoms in the energy range 13 $-500 \mathrm{eV}$ using the distorted wave method. In this study we have used a complex distortion potential as the distortion potential both at the initial and the final channels. Our results are compared amongst themselves to establish the effect of exchange and imaginary absorption potentials. We also compared with other available theoretical and experimental results to determine the suitability of our present method. From the results it is seen that when an absorption potential is included in the distortion potential, the integral cross section moves closer to the experimental result in the intermediate energy range $19-40 \mathrm{eV}$.
\end{abstract}

Key Words: Differential cross section, Integral cross section, Absorption potential, Autoionizing state, Electron impact excitation, Lambda and Alignment parameters.

\section{INTRODUCTION}

The study of electron impact excitation of autoionizing states of alkali atoms has been of interest for a long time (Roy and Rai, 1973; Nygaard, 1975; Srivastava et al., 1982; Pangantiwar and Srivastava, 1987; Borovik et al., 1999, 2005, 2008, 2009, 2011, 2012, 2013; Jobunga et al., 2012) because it can help in the explanation of the peaks observed in the ionization curves of the alkali atoms (Nygaard, 1975) and also in explaining the near threshold structures observed in the excitation-autoionization curves (Borovik et al., 2005). Some theoretical (Pangantiwar and Srivastava, 1987; Kaur and Srivastava, 1999) and experimental (Borovik et al., 2011) studies have been made on electron impact excitation of lowest autoionizing state of caesium, but no calculation on this process has been made using the distorted wave method with a complex distortion potential. In the work of Pangantiwar and Srivastava (1987) they have used the distorted wave method with a real potential (static) and Kaur and Srivastava (1999) used a relativistic distorted wave method with a real (static) potential. So, a study on electron impact excitation of the lowest autoionizing state of caesium is of interest.

In the present work we have calculated the differential and integral cross sections for electron impact excitation of the lowest autoionizing state of caesium $\left(5 p^{6} 6 s{ }^{2} S_{1 / 2}\right.$ $\left.-5 \mathrm{p}^{5} 6 \mathrm{~s}^{2} \mathrm{P}_{1 / 2,3 / 2}\right)$ in the energy range $13-500 \mathrm{eV}$ using the distorted wave method with static (real), static plus exchange (real) and static plus exchange plus absorption (complex) potentials as the distortion potentials both in the initial and final channels. We have compared our results amongst themselves to establish the effect of exchange and absorption potentials and compare our results with the theoretical results of Pangantiwar and Srivastava (1987) and Kaur and Srivastava (1999) and the experimental results of Borovik et al. (2011) to determine the suitability of our present method.

For our numerical calculation of the cross sections and alignment parameters, we have modified the Fortran computer program DWBA1 developed by Madison and Bartschat (1996) for electron- hydrogen with a real distortion potential. We have modified it for electroncaesium scattering using a complex distortion potential. 


\section{THEORY}

\section{The Distorted Wave Method}

The Hamiltonian of the electron-caesium scattering system is expressed as (Madison \& Bartschat, 1996)

$$
\mathrm{H}=\mathrm{H}_{\mathrm{o}}+\mathrm{V}
$$

where $\mathrm{H}_{\mathrm{o}}$, the unperturbed Hamiltonian describing a free motion of the electron and atom, is given by

$\mathrm{H}_{\mathrm{o}}=\mathrm{h}_{\mathrm{a}}+\mathrm{K}$

where $\mathrm{K}$ is the kinetic energy operator of the projectile electron and $h_{a}$ is the Hamiltonian of the $\mathrm{N}$-electron target atom.

$\mathrm{V}$ is the interaction potential between the projectile electron and the target atom given by the relation

$V=-\frac{Z}{r_{o}}+\sum_{i=1}^{N} \mathrm{v}_{\mathrm{i}}$

where

$\mathrm{v}_{i}=\frac{1}{r_{0 i}}$

with $\boldsymbol{r}_{o}$ the position vector of the projectile electron (taking the nucleus of the atom as the origin) and $r_{o i}$ the separation between the projectile electron and the $i^{\text {th }}$ atomic electron.

The first order T-matrix in the two potential approach is given by (Madison \& Bartschat,1996)

$$
\begin{gathered}
T_{i, f}=(N+1)\left\langle x_{f}^{-}(0) \psi_{f}(1 \ldots N)\left|\mathrm{V}-U_{f}(0)\right| A \Psi_{i}^{+}(0 \ldots \mathrm{N})\right\rangle+ \\
\left\langle x_{f}^{-}(0) \psi_{f}(1 \ldots N)\left|U_{f}(0)\right| \psi_{i}(1 \ldots N) \beta_{i}(0)\right\rangle
\end{gathered}
$$

where $\psi_{i, f}$ are the initial/final state atomic wave functions, $U_{f}$ is the final channel complex distorting potential, $A$ is the antisymmetrization operator given by

$A=\frac{1}{N+1}\left(1-\sum_{i=1}^{N} P_{i 0}\right)$

where $P_{i, 0}$ is the operator that exchanges electrons 0 and $\mathrm{i}, \beta_{i}$ is the initial state plane wave and $x_{f}^{-}$is the distorted wave in the final channel with incoming boundary conditions.

For inelastic collisions that we are considering, the second term of equation (5) vanishes due to the orthogonality of the initial and final state wave functions. We have taken the potential $U_{f}$ in equation (5) as a complex distortion potential in the final channel which includes static, exchange, and absorption potentials. This complex distortion potential is used to calculate the final distorted wave $x_{f}^{-}$by solving the Schrödinger equation $\left(\nabla_{o}^{2}+2 k_{f}^{2}-2 U_{f}\right) x_{f}^{-}=0$

The superscript - indicates incoming wave boundary conditions, $\boldsymbol{k}_{f}$ is the final state wave vector and its corresponding energy is given as $E_{f}=k_{f}^{2}$ (in atomic units).

In the first order distorted wave Born approximation, $\Psi_{i}^{+}$ in equation (5) is replaced by the product of the initial state distorted wave $x_{i}^{+}$and the initial state atomic wave function $\psi_{i}$ and then it takes the following form (for excitation process). (Expressing $\Psi_{i}^{+}$in terms of the product of an initial state distorted wave $x_{i}^{+}$times an initial state atomic wavefunction $\psi_{i}$ and making a power series expansion for the interaction potential, one gets it as the first order term.) (Madison \& Bartschat, 1996)

$T_{i, f}=(N+1)\left\langle x_{f}^{-}(0) \psi_{f}(1, \ldots N)\left|V-U_{f}(0)\right| \frac{1}{N+1}\left(1-\sum_{i=1}^{N} P_{i 0}\right) x_{i}^{+}(0) \psi_{i}(1, \ldots . N)\right\rangle$

The initial state distorted wave $x_{i}^{+}$is a solution of the Schrödinger equation

$\left(\nabla_{0}^{2}+2 k_{i}^{2}-2 U_{i}\right) x_{i}^{+}=0$

where $U_{i}$ is an arbitrary complex distorting potential in the initial channel, $k_{i}$ is the incident wave vector which is connected to the incident particle energy as $E_{i}=k_{i}^{2}$ (in atomic units).

\section{Distortion Potentials $U_{i}$ and $U_{f}$}

The distortion potential we have used is complex in nature both in initial and final channels. The complex distortion potential includes static, exchange, and absorption potentials, and is given by

$U_{i, f}=V_{i, f}^{s t}(r)+V^{e x c h}(r)+i V^{a b s}(r)$

Thus, the distortion potentials in the initial and final channels respectively are given by

$U_{i}=\left\langle\psi_{i}|V| \psi_{i}\right\rangle+V^{e x c h}(r)+i V^{a b s}(r)$

$U_{f}=\frac{1}{2}\left\langle\psi_{i}|V| \psi_{i}\right\rangle+\frac{1}{2}\left\langle\psi_{f}|V| \psi_{f}\right\rangle+V^{e x c h}(r)+i V^{a b s}(r)$

where, $V V$ is as given in equation (3) and

$V^{e x c}(r)=\frac{1}{2}\left[E-V^{s t}(r)\right]-\frac{1}{2}\left\{\left[E-V^{s t}(r)\right]^{2}+4 \pi \rho(r)\right\}^{\frac{1}{2}}$

is the exchange potential (Furness and McCarthy, 1973), $E$ is the incident particle energy, and $\rho(r)$ is the target electron charge density. The exchange potential accounts for exchange effects between the projectile electron and bound atomic electron. 
$V^{a b s}(r)=\frac{1}{2} T_{l o c} \rho(r) \sigma_{b}$

is the absorption potential (Staszewska et al., 1984), where $T_{l o c}=\left[2\left(E-V_{s e}\right)\right]^{\frac{1}{2}}$ is the local kinetic energy of the incident electron, $V_{s e}$ is the static + exchange potential and $\sigma_{b}$ is the average binary collision cross section. This absorption potential accounts for the removal of the incident projectile from the channel being considered. $V_{i}^{s t}(r)=\left\langle\psi_{i}\left|V\left(r, r_{i}\right)\right| \psi_{i}\right\rangle$ is the initial channel static potential since in the initial state the projectile only 'sees' the static potential of the target atom in its initial state. In the final state, the final state static potential is taken as $V_{f}^{s t}(r)=\frac{1}{2}\left\langle\psi_{i}\left|V\left(r_{0}, r_{i}\right)\right| \psi_{i}\right\rangle+\frac{1}{2}\left\langle\psi_{f}\left|V\left(r_{0}, r_{i}\right)\right| \psi_{f}\right\rangle$

We have taken the average of the initial and final state potential, since in the final channel, when the energy of the projectile is transferred to the atom, it takes some time (relaxation time) before the atom goes to its final state. As a result, the projectile in its final state 'sees' an intermediate potential between the initial and final state static potentials of the target.

\section{Atomic wave functions}

We have used multi zeta (MZ) wavefunctions as given by McLean and McLean (1981). They are based on the Roothan-Hartree-Fock (RHF) expansion technique. The total wave function for an N-electron atom is a Slater determinant given by

$\Psi=\frac{1}{\sqrt{N}}\left|\begin{array}{ccc}\varphi_{1}\left(\boldsymbol{r}_{1}\right) & \varphi_{2}\left(\boldsymbol{r}_{1}\right) \cdots & \varphi_{N}\left(\boldsymbol{r}_{1}\right) \\ \vdots & \vdots & \vdots \\ \varphi_{1}\left(\boldsymbol{r}_{N}\right) & \varphi_{2}\left(\boldsymbol{r}_{N}\right) \cdots & \varphi_{N}\left(\boldsymbol{r}_{N}\right)\end{array}\right|$

where,

$\varphi_{i \eta \lambda}=\sum_{p} \chi_{p \eta \lambda} C_{i \eta p}$

where $p$ refers to the $p^{\text {th }}$ basis function of symmetry $\eta$. $C_{i \eta p}$ is the expansion coefficients which depends on $\eta, p$ and $i$ but not $\lambda$.

The basic functions $\chi_{p \eta \lambda}$ are Slater-type orbitals with integer quantum numbers given as

$\chi_{p \eta \lambda}(r, \theta, \varphi)=R_{\eta p}(r) Y_{\eta \lambda}(\theta, \varphi)$

where $R_{\eta p}(r)$ is the radial Slater-type basis function given by

$R_{\eta p}(r)=N_{n} r^{n-1} e^{-\xi r}$

The normalization factor $N_{n}$, takes the form
$N_{n}=\frac{(2 \xi)^{n+\frac{1}{2}}}{\sqrt{(2 n) !}}$

\section{Evaluation of the static potentials $U_{i}$ and $U_{f}$}

The mathematical formulation of static potentials experienced by the projectile electron in the field of a caesium atom both in the initial and final channels is given as (Joachain, 1975)

$U_{s}=\left\langle\varphi_{s}|V| \varphi_{s}\right\rangle$

where $s=\mathrm{i}$ or (f) for initial or (final) state respectively. The initial atomic wave function is given as

$\varphi_{i}=\varphi(5 \mathrm{p})=\sum_{p} \chi_{i} C_{i}$

The final wave function (excited state) is given as

$\varphi_{f}=\varphi(6 s)=\Sigma_{p} \chi_{f} C_{f}$

$V$ is the interaction potential between the target atom (caesium) and the projectile (electron) and is given (in atomic units) as

$V=\left(\frac{1}{r_{0,1}}-\frac{1}{r_{0}}\right)$

treating the target as a one-electron atom.

Considering the $5 \mathrm{p}$ electron being excited to the 6 s orbital, the initial and final state static potentials are given by

$U_{i, f}=\Sigma_{n} \Sigma_{n \prime} C_{i, f} C_{i, f}\left\langle\chi_{i, f}|V| \chi_{i, f}\right\rangle$

obtained by substituting equations (21) and (22) in (20). When we substitute for the interaction potential $V$ given in equation (23) in equation (24) and expand the term $\frac{1}{r_{0,1}}$ in terms of spherical harmonics as

$\frac{1}{r_{o, 1}}=\sum_{l=0}^{\infty} \sum_{m=-1}^{+l} \frac{4 \pi}{2 l+1} \frac{r_{<}^{l}}{r_{>}^{l+1}} Y_{l, m}\left(\widehat{r}_{0}\right) Y_{l, m}^{*}\left(\widehat{r}_{1}\right)$

where $r_{<}\left(r_{>}\right)$is the lesser (greater) between $r_{0}$ and $r_{1}$, only the term associated with $l=0$ and $m=0$ contributes and since the non-spherical term contribution is very small and hence negligible (Madison et al., 1991), the static potential takes the form, after substituting $Y_{00}=\frac{1}{\sqrt{4 \pi}}$,

$U_{i, f}=2 \sum_{n} \sum_{n^{\prime}} C_{i, f} C_{i, f}\left\langle\chi_{i, f}\left|\frac{1}{r_{>}}-\frac{1}{r_{0}}\right| \chi_{i, f}\right\rangle$

Substituting the Slater-type wave function as constructed from the McLean and McLean (1981) tables in equation (22) we obtain the static potentials both in the initial and final channels as

$U_{i, f}=2 \sum_{n} \sum_{n^{\prime}} N_{n} N_{n^{\prime}} C_{n} C_{n^{\prime}} \int_{0}^{\infty} r_{1} \mu_{n}+\mu_{n^{\prime}} e^{-\left(\xi_{n}+\xi_{n} \prime\right) r_{1}}\left|\frac{1}{r_{>}}-\frac{1}{r_{0}}\right| d r$ 
where $N_{n}$ is the normalization factor, $\mu_{n}$ is the principal quantum number, $\xi_{n}$ is the orbital exponent, and $C_{n}$ is the expansion coefficient.

Splitting the integral appearing in equation (27) as $\int_{0}^{r_{0}}$ and $\int_{r_{0}}^{\infty}$, and noting that $r_{0}>r_{1}$ for the integral from 0 to $r_{0}$ and $r_{1}>r_{0}$ for the integral from $\mathrm{r}_{0}$ to $\infty$, the static potential takes the form

$U_{i, f}=2 \sum_{n} \sum_{n \prime} N_{n} N_{n^{\prime}} C_{n} C_{n \prime} \int_{r_{0}}^{\infty} r_{1}^{\mu_{n}+\mu_{n^{\prime}}} e^{-\left(\xi_{n}+\xi_{n}\right) r_{1}}\left|\frac{1}{r_{1}}-\frac{1}{r_{0}}\right| d r_{1}$

since

$$
\int_{0}^{r_{o}} r_{1}^{\mu_{n}+\mu_{n \prime}} e^{-\left(\xi_{n}+\xi_{n \prime}\right) r_{1}}\left|\frac{1}{r_{0}}-\frac{1}{r_{0}}\right| d r_{1}=0
$$

Letting $\zeta=\xi_{n}+\xi_{n \prime}$, we can write the static potential as

$V_{i, f}=2 \sum_{n} \sum_{n^{\prime}} N_{n} N_{n^{\prime}} C_{n} C_{n \prime} \int_{r_{o}}^{\infty} r_{1} \mu_{n}+\mu_{n \prime} e^{-\zeta r_{1}}\left|\frac{1}{r_{1}}-\frac{1}{r_{0}}\right| d r_{1}$

The problem reduces to analytically calculating the integrals with respect to $r_{1}$ which was done for both the initial and final states static potentials.

\section{Evaluation of direct and exchange T-matrix elements}

The system is taken as a one-electron atom excited from initial state $5 \mathrm{p}$ to final state $6 \mathrm{~s}$. After neglecting terms associated with $U_{f}(0)$ because of the orthorgonality of the atomic wave functions and orthogonality of bound and continuum wavefunctions, the transition matrices for direct and exchange processes are expressed as

$T^{\text {dir }}=\left\langle x_{f}^{-}\left(r_{o}\right) \psi_{f}\left(r_{1}\right)\left|V\left(r_{o} r_{1}\right)\right| x_{i}^{+}\left(r_{o}\right) \psi_{i}\left(r_{1}\right)\right\rangle$

$T^{e x c}=\left\langle x_{f}^{-}\left(r_{0}\right) \psi_{f}\left(r_{1}\right)\left|V\left(r_{o} r_{1}\right)\right| x_{i}^{+}\left(r_{1}\right) \psi_{i}\left(r_{0}\right)\right\rangle$

where $V\left(r_{0} r_{1}\right)=\left(\frac{1}{r_{01}}-\frac{1}{r_{0}}\right)$ is the interaction potential between the projectile electron and target atom. To evaluate the scattering amplitudes (30) and (31), the distorted waves $x_{i}^{+}$and $x_{f}^{-}$are expanded in terms of partial waves (Singh, 2004; Madison and Bartschart, 1996), giving

$$
\begin{aligned}
& \mid x_{i}^{+}>=\sqrt{\frac{2}{\pi}} \frac{1}{k_{i} r} \sum_{l_{i} m_{i}} i^{l_{i}} x_{l_{i}}\left(k_{i}, r\right) Y_{l_{i} m_{i}}(r) Y_{l_{i} m_{i}}^{*}\left(k_{i}\right) \\
& \mid x_{f}^{-}>=\sqrt{\frac{2}{\pi}} \frac{1}{k_{f} r} \sum_{l_{f} m_{f}} i^{l_{f}} x_{l_{f}}^{*}\left(k_{f} r\right) Y_{l_{f} m_{f}}(r) Y_{l_{f} m_{f}}^{*}\left(k_{f}\right) .
\end{aligned}
$$

Substituting (32) and (33) in the Schrodinger equations (9) and (7) respectively we obtain radial distorted waves $x_{l_{i}}, x_{l_{f}}$ which are the solutions of the equation

$x_{l_{s}}^{\prime \prime}(r)+\left[k_{s}^{2}-\frac{l_{s}\left(l_{s}+1\right)}{r^{2}}-U_{s}(r)\right] x_{l_{s}}(r)=0$

where $s=i, f$.

We have calculated the differential cross section summed over magnetic sub levels using the formula

$\left(\frac{d \sigma}{d \Omega}\right)_{5 p \rightarrow 6 s}=(4 \pi)^{4} \frac{k_{f}}{k_{i}}\left[\frac{1}{4}\left|T_{5 p \rightarrow 6 s}^{d i r}+T_{5 p \rightarrow 6 s}^{8 x c h}\right|^{2}+\frac{3}{4}\left|T_{5 p \rightarrow 6 s}^{d i r}-T_{5 p \rightarrow 6 s}^{8 x c h}\right|^{2}\right]$

The integral or total cross section is obtained by summing up the differential cross section given by the relation

$\sigma=\int_{0}^{2 \pi} \int_{0}^{\pi} \sin \theta \frac{d \sigma}{d \Omega} d \theta d \varphi$.

We have also calculated $\lambda$ and alignment parameter $A_{20}$ using the relations

$\lambda=\frac{\sigma_{0}(\theta, \varphi)}{\sigma_{0}(\theta, \varphi)+2 \sigma_{1}(\theta, \varphi)} 0 \leq \lambda \leq 1$

where $\sigma_{0}(\theta, \varphi)$ and $\sigma_{1}(\theta, \varphi)$ are the differential cross sections for $n p_{m} \rightarrow(n+1) s$ transition where $\mathrm{m}=0$ or 1 , and

$A_{20}=\frac{\sigma_{1}-\sigma_{0}}{\sigma}$

where $\sigma_{0}$ and $\sigma_{1}$ are integral cross sections for magnetic sublevels $m=0$ and $m=1$ respectively.

\section{RESULTS AND DISCUSSION}

We have calculated the differential and integral cross sections and angular correlation parameters for electroncaesium scattering using a complex distortion potential both in the initial and final channels. The impact energy ranges between $13-500 \mathrm{eV}$. Our results are shown in Figure 1 for integral cross section, in Figures $2-7$ for differential cross section, in Figure 8 for the alignment parameter and in Figures $9-12$ for $\lambda$ parameters.

In Figure 1 the present integral cross section results obtained using static potential and static plus absorption potential experience a sharp increase in the cross section just around the excitation threshold $(12.9 \mathrm{eV})$ (negative ion resonances), a phenomenon that is attributed to the formation of a negative ion when the projectile electron is left with very little energy after exciting the target atom. When we include the absorption potential (Staszewska et al., 1984), the results are brought closer to the experimental results between $19-40 \mathrm{eV}$. 


\section{Integral cross section}

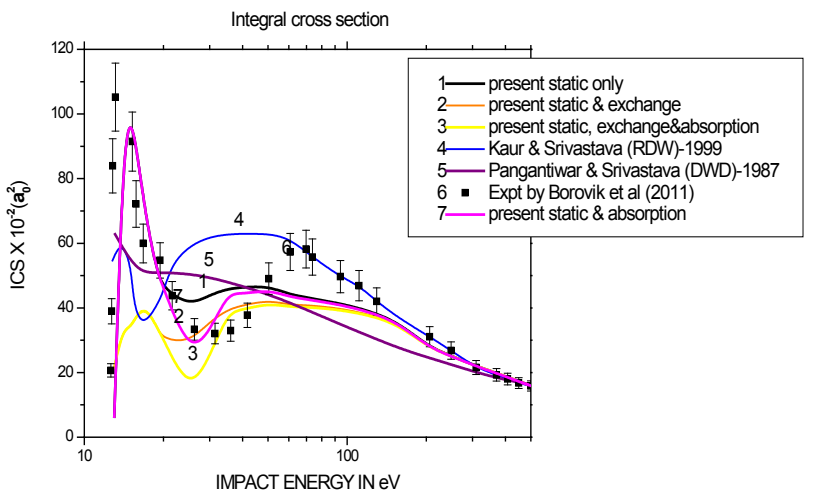

Figure 1. Integral cross section results for electron impact excitation of the lowest autoionizing state of caesium using different distortion potentials (static only; static plus exchange; static plus absorption; static plus exchange plus absorption).

\section{Differential cross section}

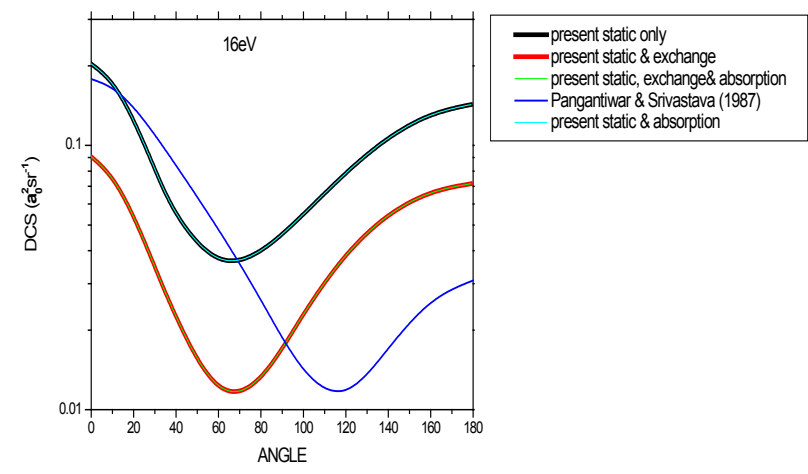

Figure 2. Differential cross section results for electron impact excitation of the lowest autoionizing state of caesium at 16 $\mathrm{eV}$ using different distortion potentials (static only; static plus exchange; static plus absorption; static plus exchange plus absorption). The graph of our present static plus absorption potential is mounted on the graph of our present static only potential while the graph of our present static, exchange plus absorption potential is mounted on the graph of our present static plus exchange potential.

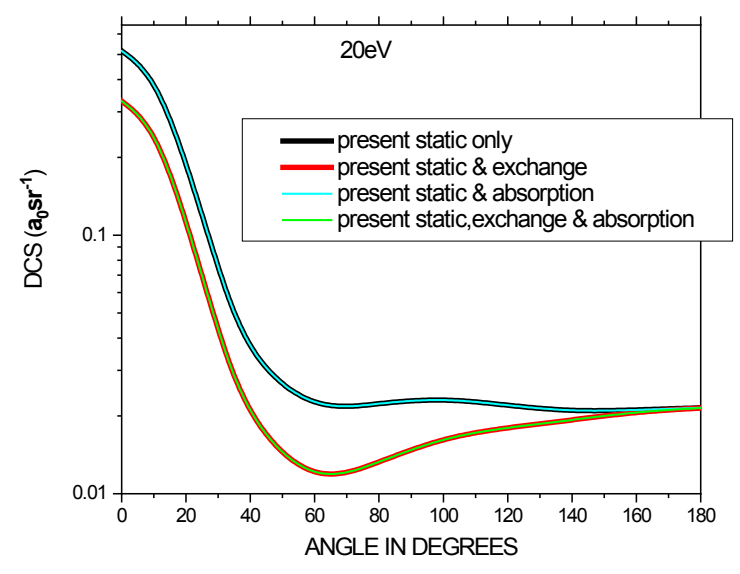

Figure 3. Differential cross section results for electron impact excitation of the lowest autoionizing state of caesium at 20 $\mathrm{eV}$ using different distortion potentials (static only; static plus exchange; static plus absorption; static plus exchange plus absorption). The graph of our present static plus absorption potential is mounted on the graph of our present static only potential while the graph of present static, exchange plus absorption potential is mounted on the graph of our present static plus exchange potential.

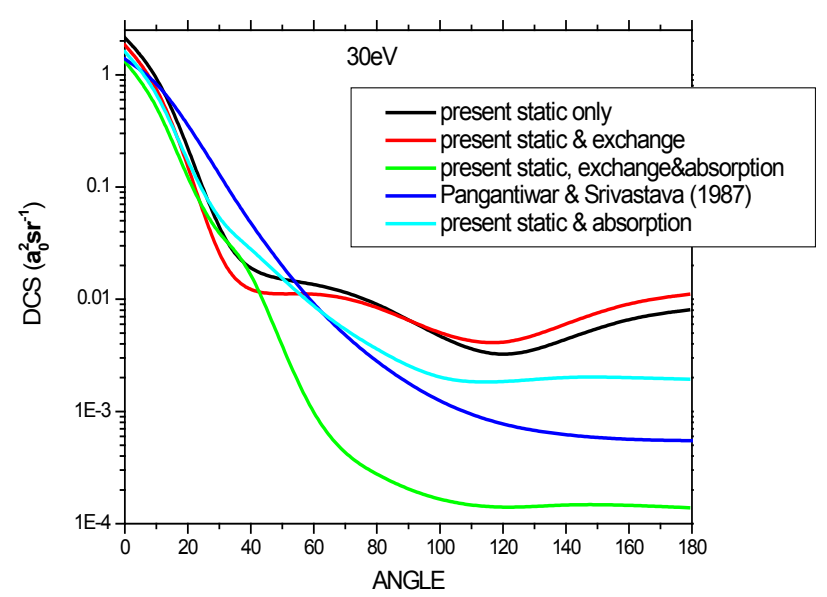

Figure 4. Differential cross section results for electron impact excitation of the lowest autoionizing state of caesium at 30 $\mathrm{eV}$ using different distortion potentials (static only; static plus exchange; static plus absorption; static plus exchange plus absorption).

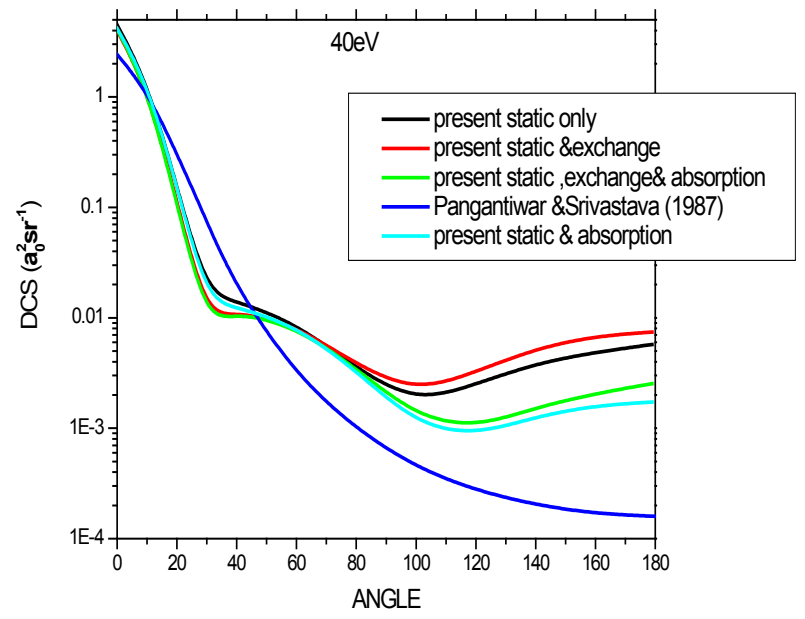

Figure 5. Differential cross section results for electron impact excitation of the lowest autoionizing state of caesium at 40 $\mathrm{eV}$ using different distortion potentials (static only; static plus exchange; static plus absorption; static plus exchange plus absorption).

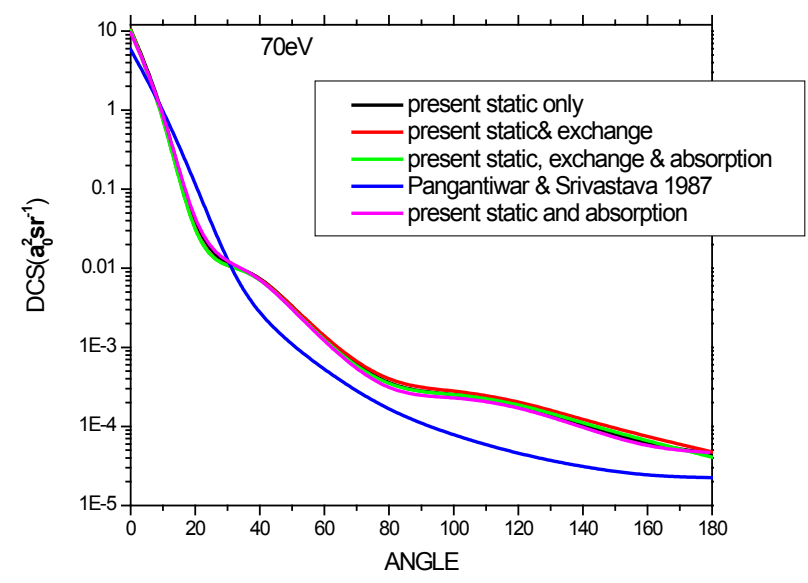

Figure 6. Differential cross section results for electron impact excitation of the lowest autoionizing state of caesium at 70 $\mathrm{eV}$ using different distortion potentials (static only; static plus exchange; static plus absorption; static plus exchange plus absorption). 
Figures $2-7$ present the differential cross section (DCS) results at different electron impact energies. The DCSs have maximum value at small scattering angles and they reduce as the angles increase except at very low impact energy. This means that more particles are scattered at small scattering angles and less at large angles. As the projectile energy increases, the particle encounters less interaction with the target atom, hence it is scattered at a small angle. The inclusion of the exchange and absorption potential lowers the DCS across the energy range of the study but does not change the trend. As the impact energy increases, the agreement between our present results improves. This is because as the impact energy increases, the difference in the distortion potentials become less important. At lower impact energies, the absorption potential has little effect on the DCS. This is because very few other channels are open at this energy. That explains why the inclusion of an absorption potential at impact energies $16 \mathrm{eV}$ and $20 \mathrm{eV}$ (Figures 2 and 3) does not change the DCS at these energies. But as the impact energy increases, more channels are open and the inclusion of absorption potential shows its effect as we see at 30 and $40 \mathrm{eV}$ electron impact energies. The present DCS results are in qualitative agreement amongst themselves and with those of Pangantiwar and Srivastava (1987) at all impact energies considered. Though there are few discrepancies which are attributed to different distortion potentials used at both the initial and final channels and also the different method of calculation of the transition matrix.

Our alignment parameter results for static and static plus absorption as the distortion potentials are in very close agreement with the relativistic distorted wave (without exchange) results (RDWD) of Kaur and Srivastava (1999). From the formula of alignment parameter, when $A_{20}$ is negative indicates that $\boldsymbol{\sigma}_{0}$ is greater than $\boldsymbol{\sigma}_{1}$. This means that most of the excitations are taking place from the magnetic substate $\mathrm{m}=0$ and when $\mathrm{A}_{20}$ is positive, it means that $\boldsymbol{\sigma}_{1}$ is greater than $\boldsymbol{\sigma}_{0}$ and most of the excitations are taking place from $m=1$ magnetic substate. In our study, Figure 8, from just above excitation threshold up to around $350 \mathrm{eV}$, the alignment parameter is negative indicating that most of the incident electrons are scattered from the magnetic substate $m=0$, compared to magnetic substate $\mathrm{m}=1$. Inclusion of absorption potentials slightly reduces the alignment parameter across the projectile impact energy range. Further inclusion of exchange potentials gives a slight reduction of the alignment parameter at intermediate energy range. Between $60-150 \mathrm{eV}$, our results for static and static plus absorption as the distortion potentials are in perfect agreement with the results of Kaur and Srivastava (1999).

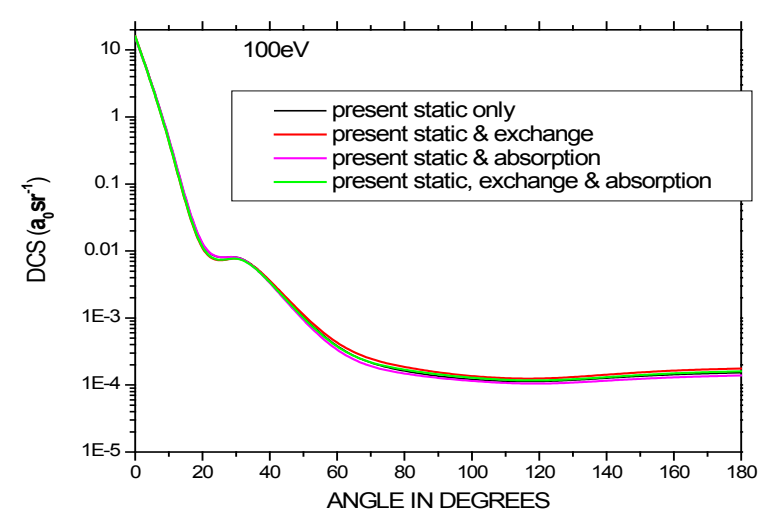

Figure 7. Differential cross section results for electron impact excitation of the lowest autoionizing state of caesium at 100 $\mathrm{eV}$ using different distortion potentials (static only; static plus exchange; static plus absorption; static plus exchange plus absorption).

\section{Alignment parameter}

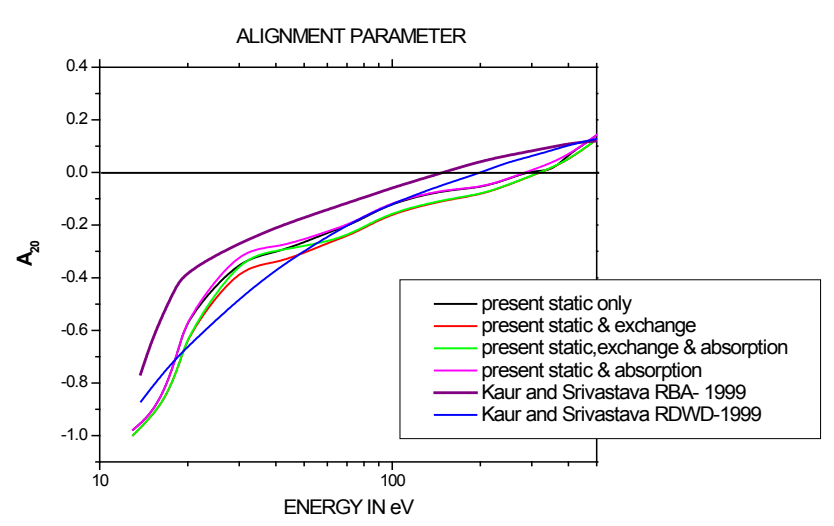

Figure 8. Alignment parameter results for electron impact excitation of the lowest autoionizing state of caesium using different distortion potentials (static only; static plus exchange; static plus absorption; static plus exchange plus absorption).

\section{Lambda parameter}

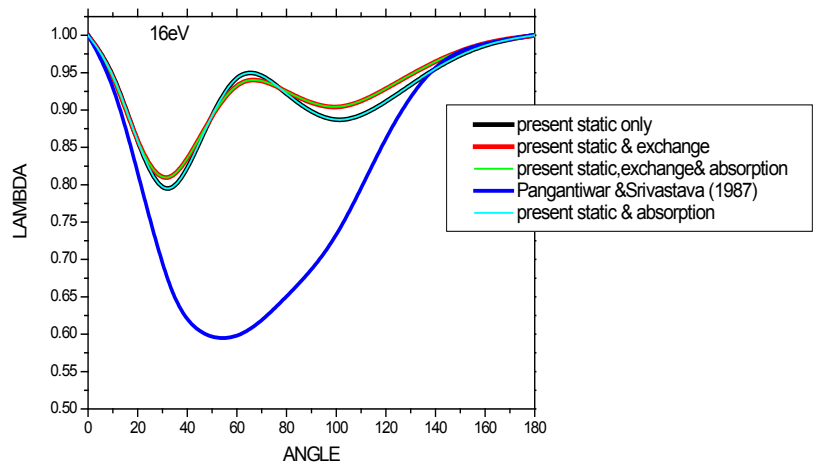

Figure 9. $\lambda$ parameter results for electron impact excitation of the lowest autoionizing state of caesium at $16 \mathrm{eV}$ using different distortion potentials (static only; static plus exchange; static plus absorption; static plus exchange plus absorption). The graph of present static plus absorption potential is mounted on the graph of our present static only potential, while the graph of our present static, exchange plus absorption potential is mounted on the graph of our present static plus exchange potential. 


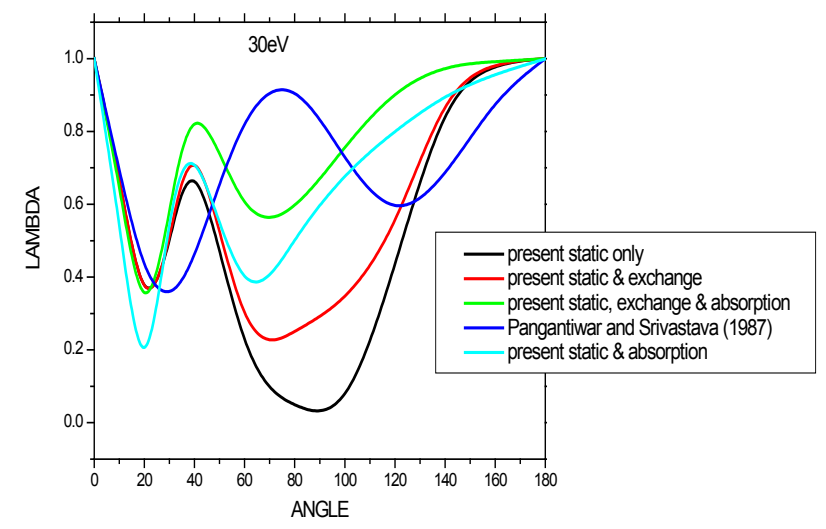

Figure 10. $\lambda$ parameter results for electron impact excitation of the lowest autoionizing state of caesium at $30 \mathrm{eV}$ using different distortion potentials (static only; static plus exchange; static plus absorption; static plus exchange plus absorption).

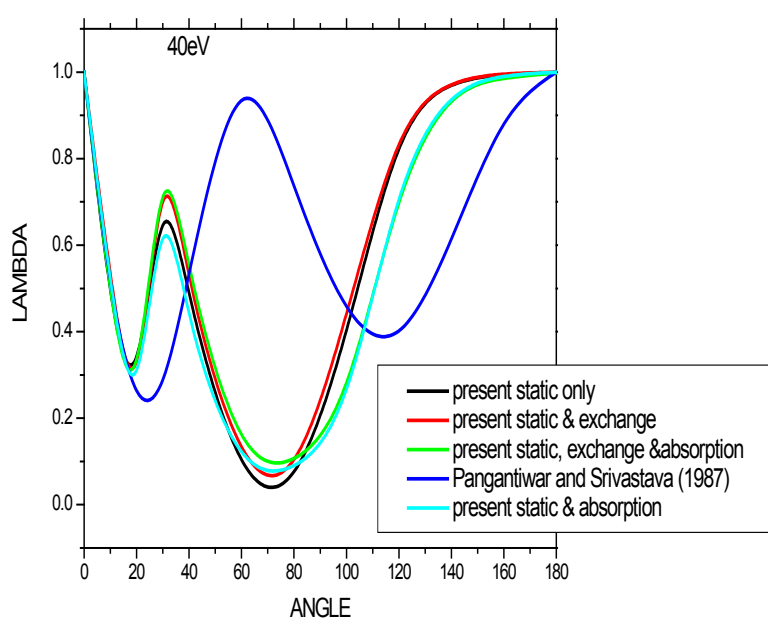

Figure 11. $\lambda$ parameter results for electron impact excitation of the lowest autoionizing state of caesium at $40 \mathrm{eV}$ using different distortion potentials (static only; static plus exchange; static plus absorption; static plus exchange plus absorption.

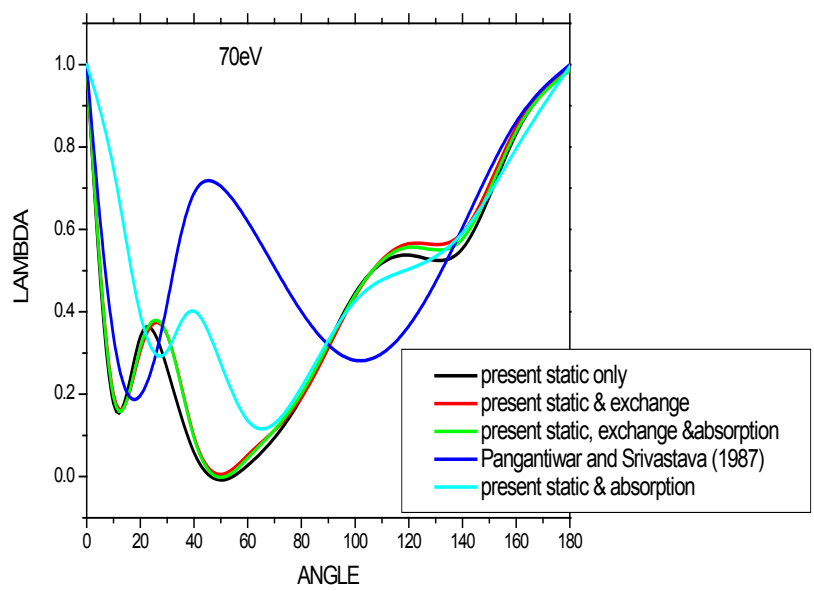

Figure 12. $\lambda$ parameter results for electron impact excitation of the lowest autoionizing state of caesium at $70 \mathrm{eV}$ using different distortion potentials (static only; static plus exchange; static plus absorption; static plus exchange plus absorption).
Our results show that most of the particles are scattered from the magnetic substate $\mathrm{m}=0$ as indicated in lower and upper scattering angles in our present lambda parameter results for all the impact energies. We see from Figures $9-$ 12 that as the impact energy increases most of the particles scattered at intermediate angles are scattered from $\mathrm{m}=1$ magnetic sublevel, since $\lambda<0.3$. When $\lambda=0.3$, then the particles are evenly scattered from the two states.

At $16 \mathrm{eV}$ which is closer to the excitation energy, our results are in perfect agreement with the results of Pangantiwar and Srivastava (1987) at lower and upper scattering angles, with disparity at intermediate scattering angles $\left(30^{\circ}-140^{\circ}\right)$. At 30, 40 and $70 \mathrm{eV}$, our present $\lambda$ results are in good qualitative and quantitative agreement at lower scattering angles, while in upper scattering angles there is disparity which is associated to different distortion potential used in the initial and final channels. Also, in the present study we have calculated the $\lambda$ parameter for the unresolved state while Pangantiwar and Srivastava (1987) studied the $\lambda$ parameter only for the fine structure state $\left({ }^{2} P_{\frac{s}{2}}\right)$ of the caesium atom.

\section{CONCLUSION}

In conclusion our present results for integral cross section compares well with the experimental results of Borovik et al. (2011) around excitation threshold when we use real distortion potentials but with disparity at the intermediate energy region. When the imaginary absorption potential (Staszewska et al., 1984) is included, the results are improved at the intermediate energies (our present results are brought closer to the experimental results). The exchange potential drastically reduces the integral cross section around the excitation threshold (Furness \& McCarthy, 1973). The change is very big, and this suggests that this model of exchange potential is not suitable for heavy alkali metals such as caesium. The present DCS results are in qualitative agreement with those of Pangantiwar and Srivastava (1987) at all impact energies considered. Though there are few discrepancies which are attributed to different distortion potentials used in both the initial and final channels, the present alignment parameter results are in good qualitative agreement with the theoretical work of Kaur and Srivastava (1999). It is seen from the present results for the $\lambda$ parameter that, as the impact energy increases, most of the particles scattered at intermediate angles are scattered from the $\mathrm{m}=1$ substate.

\section{ACKNOWLEDGEMENT}

We thank the Department of Physics, Kenyatta University for providing the facility to work on this project. 


\section{REFERENCES}

Borovik, A. A., Rojas, H. L., Kings, G. C., \& Remeta, E. Y. (1999). Electron-impact excitation of potassium autoionizing levels in the near-threshold region. Journal of Physics B: Atomic, Molecular and Optical Physics, 32, 4225 .

Borovik, A. A., Grum-Grzhimailo, A. N., Bartschat, K., \& Zatsarinny, O. (2005). Electron impact excitation of the $\left(3 \mathrm{p}^{5} 4 \mathrm{~s}^{2}\right)^{2} \mathrm{P}_{3 / 2,1 / 2}$ autoionizing states in potassium. Journal of Physics B: Atomic, Molecular and Optical Physics, 38, 1081.

Borovik, A. A., Zatsarinny, O., \& Bartschat, K. (2008). Near-threshold electron-impact excitation of the $\left(2 \mathrm{p}^{5} 3 \mathrm{~s}^{2}\right)^{2} \mathrm{P}_{3 / 2,1 / 2}$ autoionizing states in sodium. Journal of Physics B: Atomic, Molecular and Optical Physics, 41, 035206.

Borovik, A. A., Zatsarinny, O., \& Bartschat, K. (2009). Resonance effects in electron and photon impact excitation of the $\mathrm{p}^{6}$ subvalence subshell in alkali atoms. Journal of Physics B: Atomic, Molecular and Optical Physics, 42, 044010.

Borovik, A. A., Kupliauskiene, A., \& Zatsarinny, O. (2011). Excitation cross sections and spectroscopic classification of autoionizing levels in a caesium atom. Journal of Physics B: Atomic, Molecular and Optical Physics, 44, 145203.

Borovik, A. A., Roman, V., \& Kupliauskiene, A. (2012). The $4 \mathrm{p}^{6}$ autoionization cross section of $\mathrm{Rb}$ atoms excited by low energy electron impact. Journal of Physics B: Atomic, Molecular and Optical Physics, 45, 045204.

Borovik, A. A., Roman, V, Zatsarinny, O., \& Bartschat, K. (2013). Electron impact excitation of the lowest doublet and quartet core-excited autoionizing states in $\mathrm{Rb}$ atoms. Journal of Physics B: Atomic, Molecular and Optical Physics, 46, 015203.

Furness, J. B., \& McCarthy, I. E. (1973). Semi phenomenological optical model for the electro scattering on atoms. Journal of Physics B: Atomic and Molecular physics, 6, 2280.

Joachain, C. J. (1975). Quantum Collision Theory. North Holland Publishing Company, Amsterdam. Pp. 3-11, 442-621.

Jobunga, E., Okumu, J., \& Singh, C. S. (2012). Excitation cross section evaluation for the lowest autoionizing state of potassium. African Review of Physics, 7, 0001.

Kaur, S., \& Srivastava, R. (1999). Excitation of the lowest autoionizing $n p^{5}(\mathrm{n}+1) \mathrm{s}^{2},{ }^{2} P_{\frac{\mathrm{s}}{2}}, \frac{1}{2}$ states of $\mathrm{Na}(\mathrm{n}=2), \mathrm{K}$ $(\mathrm{n}=3), \mathrm{Rb}(\mathrm{n}=4)$ and $\mathrm{Cs}(\mathrm{n}=5)$ by electron impact. Journal of Physics B: Atomic, Molecular and Optical Physics, 32, 2323.

Madison, D. H., \& Barschart, K. (1996). The distorted wave method for elastic scattering and atomic excitations. In Computational Atomic Physics Ed. K. Bartschat. Springer Verlag, Berlin.

Madison, D. H., Bartschat, K., \& Peacher, J. L. (1991). Effect of non-spherical distorting potentials in a firstorder distorted-wave calculation. Physical Review A, 44, 304.

McLean, A. D., \& McLean, R. S. (1981). RoothanHartree-Fock Atomic wave functions: Slater -Basis-Set Expansions for $\mathrm{Z}=55-92$. Atomic Data and Nuclear Tables, 26, 197.

Nygaard, K. J. (1975). Electron impact autoionization in heavy alkali metals. Journal of Physics B: Atomic and Molecular Physics, 11, 1475.

Pangantiwar, A. W., \& Srivastava, R. (1987). $e^{ \pm}$impact excitation of the autoionizing levels in alkalis: a distorted wave approach. Journal of Physics B: Atomic and molecular Physics, 20, 5881.

Roy, B. N., \& Rai, D. K. (1973). Electron-impact ionization of alkali metals. Physical Review A, 8, 849.

Singh, C. S. (2004). Magnetic sublevel differential cross sections for electron impact of $2^{1} \mathrm{P}$ of Helium. East African Journal of physical sciences, 5, 85.

Srivastava, R., Singh, C. S., \& Rai, D. K. (1982). Excitation of the lowest autoionizing states in alkalis. Journal of Physics B: Atomic and Molecular Physics, 15, 1899.

Staszewska, G., Schwenke, D. W., \& Truhlar, G.D. (1984). Investigation of the shape of the imaginary part of the optical-model potential for electron scattering by rare gases. Physical Review A, 29, 3078. 